\title{
Implementation and Improvement of Solar Power Data Monitoring and Sharing Platform based on IPv6
}

\author{
Guojing Zhang ${ }^{1}$, Xiaoying Wang ${ }^{1+}$ and Yuling $\mathrm{Li}^{3}$ \\ Department of Computer Technology and Applications \\ Qinghai University, Xining, China, 810016
}

\begin{abstract}
As the application of photovoltaic power station system becomes more and more widely, research and implementation of the photovoltaic power station data display and sharing platform plays a significant role for related research. In this paper, we designed, implemented and improved the IPv6-based solar data monitoring and sharing platform. In this system, the function of real-time data updating was realized and improved. To improve the original system, we added the functions of data processing and statistical analysis, and modified the data display interface and user management functions. In order to establish the IPv6 photovoltaic power station data sharing and display platform, we used the B/S architecture to present and display the real-time data collected by the solar power station in the form of Web to realize data sharing. The system could provide online data sharing supporting IPv6 protocol through functional modules such as remote data synchronization and foreground asynchronous display.
\end{abstract}

Keywords: photovoltaic power station; monitoring data; statistical analysis; data processing

\section{Introduction}

At present, the environmental pollution caused by China's traditional high-pollution and high energy consuming economic growth model is increasingly prominent. In order to ensure the stable and efficient operation of solar power generation equipment, the real-time monitoring data in the solar panel power generation process must be effectively transmitted to the solar power generation monitoring system, so as to be able to timely and effectively understand the working environment and situation of the monitored solar power generation array [1]. Yu et al. [2] proposed a technical and economic indicator model for evaluating system performance.Research on solar photovoltaic power generation has also been widely conducted in other countries. F.S. Abdullah et al. [3] designed a solar tracker, which can receive solar radiation and produce maximum power through a photovoltaic panel (PV) system. Park et al. [4] designed a power generation device and a diagnostic solar photovoltaic power generation monitoring system by comparing estimated power generation with actual power. S. A. Bogachev et al. [5] designed a solar photovoltaic power generation monitoring and control system for the characteristics of solar photovoltaic power plants. Kalogirou et al.[6]aimed to give a survey of possible solutions of PV and STS integration on the building roofs and facaeds. The advantages of integration are quantified and suggestions are given to address the possible problems created. Trieb et al.[7] combining the large demand of clean electricity in Europe (EU) with the large potential of solar electricity generation from concentrating solar powerstations (CSP) in the Middle East and North Africa (MENA) can provide both climate protection and development for both regions and lead to environmental, economical and social sustainability. Benson et al.[8] analyzes two pairs of renewable energy technologies and fnds that the annual improvement rate of cost/investment is quite different for the four technological domains.

\footnotetext{
+ Corresponding author. Tel.: 13734603501
}

E-mail address: 10853243@qq.com 
In this paper, the system we implemented is an IPv6-based solar data monitoring and sharing platform. We improved the system from various aspects, including modifying the interface, adding new features, optimizing the real-time data update process, and adding a part of the data processing to make the site. The system features became more complete and the data display interface could be better exhibited. By optimizing the website system, it could provide convenient sharing and analysis of solar energy data resources for scientific researchers on campus, providing a platform for users to share the solar energy platform as a data resource center for downloading.

\section{System Architecture}

\subsection{Architecture design of the photovoltaic power plant monitoring system}

The main purpose of this paper is to improve the previous system we have implemented preliminarily [9], so the details of the overall structure of the PV power plant is omitted in this paper. The photovoltaic power station data sharing and display platform designed is based on the small solar photovoltaic power station currently set up by the Department of Computer Technology and Applications of Qinghai University. The power station could collect a series of data related to solar power generation equipment and environmental monitoring data on a regular basis, but these data are difficult to obtain in a convenient and timely manner, and are not conducive to visual inspection. Therefore, in view of the above situation, this paper optimizes the data synchronization method, increases the data analysis function, and the user login and registration part based on the original PV power plant monitoring data sharing platform. This paper optimizes the function of the solar monitoring system, which is more comprehensive and more convenient for users to share the solar data.

\subsection{Architecture design of Web data sharing system}

Figure 1 shows the architecture design of an IPv6-based data sharing system. The data sharing system architecture is divided into three layers, including the database layer, the business logic layer, and the Web presentation layer. The database layer uses MySQL database to analyze and design the system's functional modules. The business logic layer uses JSP (Java Server Pages) Servlet technology to analyze and design the system's functional modules. The Web presentation layer uses JSP technology to dynamically generate Web pages. The JSP is executed on the server side, and returns an HTML text to the client, so the client can browse as long as there is a browser. When the web server encounters a request to access a JSP page, it first executes the JSP program and then returns the result to the client along with the HTML code in the file.

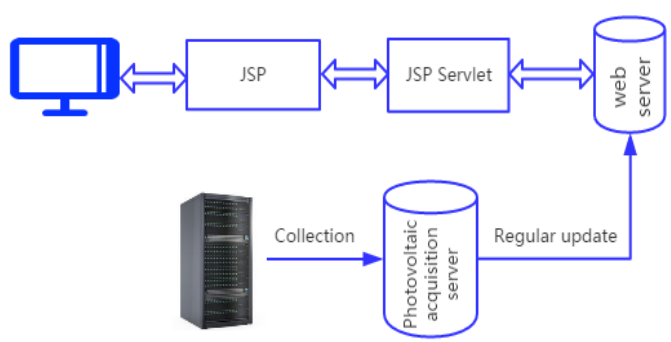

Fig. 1: Data sharing system architecture design based on IPv6

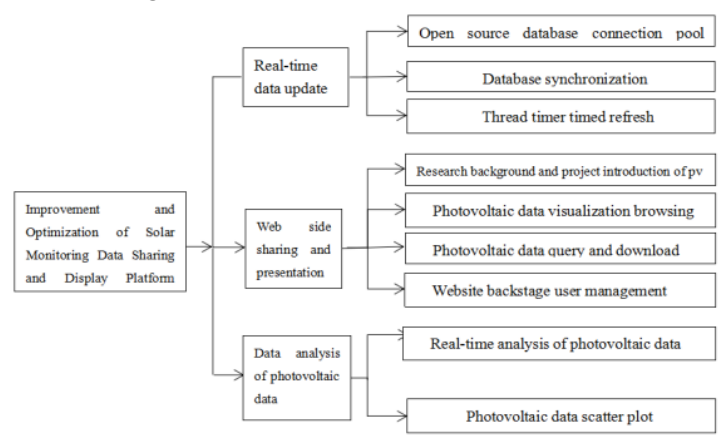

Fig. 2: System function structure

\subsection{Function design of the Web data sharing system}

The current system function of the system is divided into three main parts, the first part is the front-end display, the second part is the back-end data update and data synchronization, and the third part is data processing. The front-end display mainly contains the project profile and photovoltaic research background, online display, data download and data statistics analysis, website background management display interface. The back-end is the data update and data synchronization, where the data update process is use the database connection pool Druid to update the data to the database on the website server. The online display interface shows the output voltage, output current, output power, power consumption, illuminance, wind speed, and temperature variation over different time periods, where the data is updated every ten minutes. The statistical 
analysis interface uses the histogram of Hellochart to analyze the maximum, minimum, average and standard deviation of the power supply every day, and shows the relationship between the illuminance and the amount of electricity generated of one day, and the relationship between the illuminance and the wind speed. The system function structure is shown in Fig.2.

\section{Database Design And Data Processing}

\subsection{Data synchronization optimization}

Since the previous PV acquisition system stores the collected PV data in the EXCEL data table every ten minutes, so the data must be synchronized to the website server and then read into the MySQL database. The data synchronization is to use the free data real-time synchronization software freefilesync and realtimesync to synchronize the local data to the database of website server. We use the java language to write the data reading program, and package it into Excel-mysql.jar program to write data to the MySQL database. Since recently we replaced the PV acquisition system, we had to modify the real-time data synchronization method according to the requirements. The updated PV collection system stores the collected PV data directly in the MySQL database of the server where the system is located every ten minutes, and the PV collection system will collect the data for the abnormal data rejection and screening process before writing to the database. Considering that the long-term PV data collection requires a large storage space, we allocate the PV collection server and the website display server to different machines during deployment. In the new system, we use database connection pool Druid provided by Alibaba for real-time data synchronization. The data synchronous comparison chart of original and new system is shown in Fig.3.

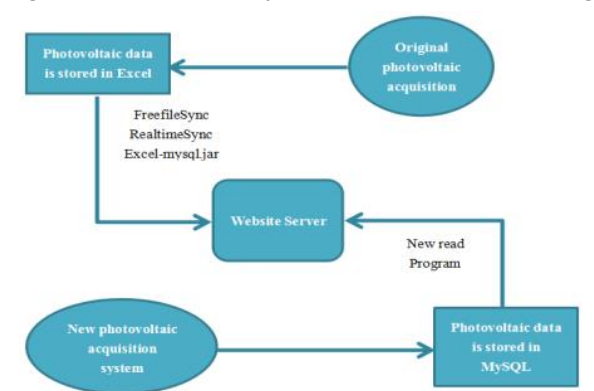

Fig. 3: Photovoltaic data synchronization comparison

\subsection{Data synchronization process}

In our system, we used the Alibaba open source database connection pool called Druid and Spring JTemplete to write real-time data synchronization programs in Java. Druid is currently the best database connection pool, in terms of functionality, performance, and scalability, and it surpasses other database connection pools, including DBCP, C3P0, BoneCP, Proxool, and JBoss DataSource. Druid is a JDBC component and its advantages mainly include: 1) efficient, powerful, and scalable; 2) can monitor database access performance; 3 ) can provide database password encryption。

In this system, the PV data acquisition system performs data collection every ten minutes, as shown in Fig. 4. So in the data synchronization, this new system uses Java multi-thread to implement an inner loop of while(1), which is used to set the timer Thread.sleep $(10 * 60 * 1000)$ to refresh the data every ten minutes. The data in the PV collection database software is imported into the database solarenergy every ten minutes, where the database solarenergy and software are located at the server with IP 49.209.80.36 and 49.209.80.151, respectively.

\section{Implementation steps:}

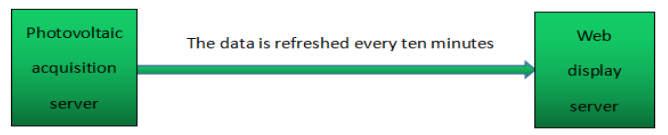

Fig. 4: Data refresh time diagram

1. The encapsulation class of the implementation data is saved under the domain package, and the type of the data is set. If the data type cannot be inserted, the conversion is performed using SimpleDateFormat. 
2. Create two new druid.properties files, configure the IP address of the two databases, the database name, and the user password. If garbled characters appear, we will use utf- 8 for encoding.

3. The database import is implemented in the database directory. In the process of synchronizing the PV data, the data type of all kinds of data must be kept consistent with the data type in the original database. Otherwise a format conversion error will occur.

4. The main class implements the runnable interface, implements a multi-threaded operation to achieve an inner loop of while(1), and is used to periodically refresh the data every ten minutes.

\subsection{Statistical Analysis of Data}

Relevance analysis refers to the analysis of two or more variables with correlation, so as to measure the degree of correlation between the two variables. In this paper, in order to intuitively show the relationship between the daily photovoltaic power generation, illumination and wind speed, in the system we added the data statistical analysis function module on the basis of the original system. In this module, the main content of statistical analysis page is the data analysis interface for the electrical parameters and environmental parameters. Here we use the Highcharts library, which is a pure JavaScript library and can easily add interactive feature to the web site. The initial data is stored in different data tables, and the relationship between correlation values and illumination is mainly discussed. The corresponding data is obtained through a fixed time field. Power generation is stored in the data sheet in an accumulative manner and is cleared to zero every day. Therefore, in order to obtain the corresponding data at each time, the power generation at each time is calculated by using the descending method, and the starting power is calculated by the time ratio, and then the scatter diagram is formed by the relationship between the light intensity and the corresponding time. Similarly, the corresponding $j s$ function is called to display the required data according to the users' clicks. In the correlation analysis, we also show that the correlation functions in $j s$ file are called to process the obtained data and visualize the data.

\section{Implementation of Data Display Interface}

\subsection{Home page interface}

The navigation bar in the home page includes: home page, project introduction, online display, statistical analysis, contact us, user login, download resources, background management. The original system did not have a statistical analysis interface, so in this paper we added the function of statistical analysis. The homepage of the website is shown in Fig.5.

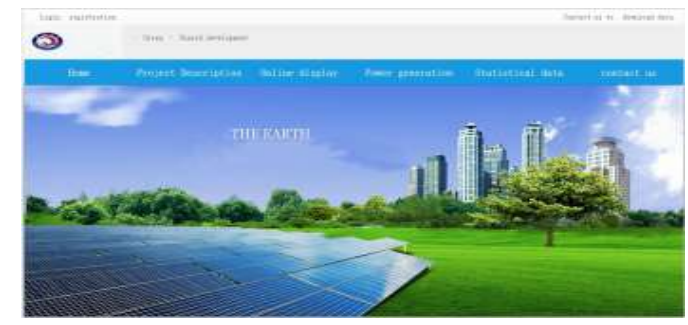

Fig. 5: The home page

\subsection{Online display interface}

This website calls Highcharts to show the data graph. According to the particularity of data, this website selects a line chart with a scalable time axis. Online display mainly refers to the time period and parameters selected by the user. According to the users' choices, the front end returns the users' requests and queries the data from the database. Line charts are used to display the monitoring data. The data from the starting time 2018-01-01 to the ending time 2018-01-07 are displayed by default. However, the users can zoom in to query more detailed data according to their own needs. The online display page is shown in Fig. 6. 


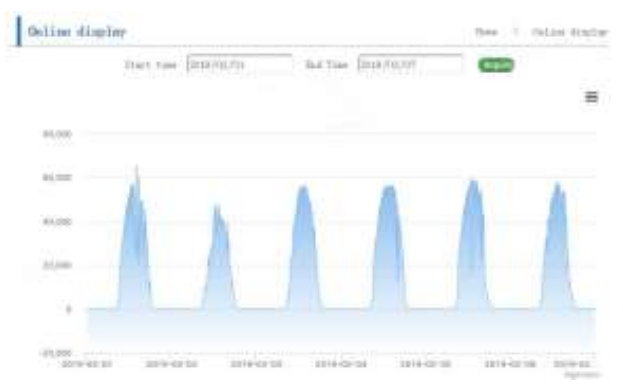

Fig. 6: Online display interface diagram

\subsection{Statistical Analysis Interface}

Fig. 7 is a web page showing the statistical analysis of photovoltaic data. According to the selected time period, this page can display the maximum value, minimum value, average value and standard deviation of power supply power under the current date.
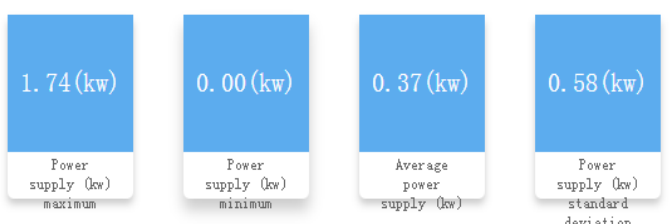

Fig. 7: Photovoltaic data statistical analysis page

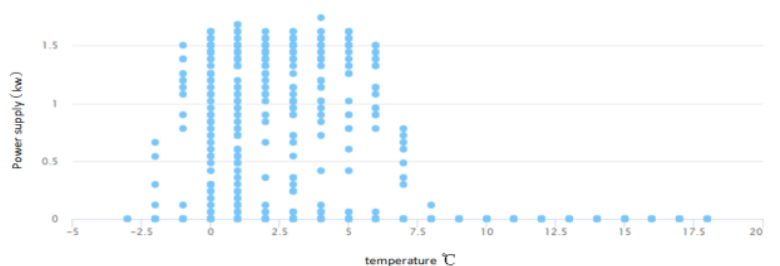

Fig. 8: Hash relation diagram of power supply power and illumination intensity

Figure 8 is a scatter plot of the power supply of commercial power parameters and the illumination intensity of environmental parameters. It is obvious from the diagram that the higher the illumination intensity is, the higher the power supply will be. This implies that the illumination intensity is proportional to the power supply amount.

\subsection{IPv6 Support}

To enable this system to support IPv6 access, the following Settings are required.

- Connect the Web Server to campus network backbone supporting IPv6.

- Set up the IPv6 address, subnet mask, and gateway for the Web server correctly.

The following changes are made in the Tomcat service configuration file:

First, Check whether IPV6 is supported successfully using "ipconfig /all". If the local IPv6 address "e80::d423:34af:7a7c:c995\%10 " in the output, it means that IPv6 was already opened.

Second, modify the Tomcat configuration file. A line of code has to be added into the configuration file, as shown in bold fonts in Fig.9.

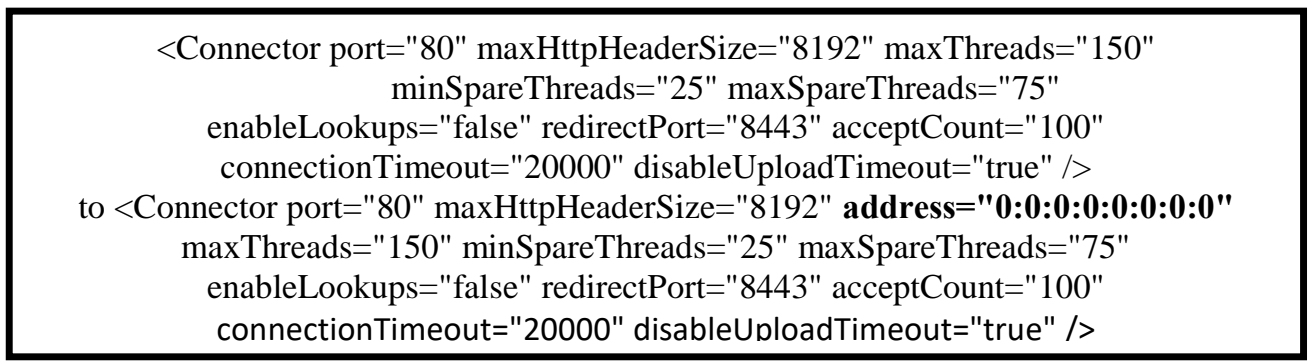

Fig. 9: Part of Tomcat configuration file

Thirdly, restart Tomcat to view the listener.

- After the deployment, this website can be accessed directly through IPv6 address. The IPv6 address is of our website is:

http://[2001:250:1e01:b:65f6:aae1:56dc:2ec2]:11115/Power/.

\section{Conclusion}


The system has been implemented and improved on the basis of the previously deployed platform, making full use of the advantages of the original web-based solar monitoring data sharing platform. The current system has better interface, more efficient way of data updating, and the addition of the functions of removing and filtering abnormal data and statistical analysis, thus making the system function easier to use. The system platform provides a window for schools to display the achievements of using photovoltaic energy, and opens up a good channel for promoting the utilization of solar energy.

\section{Acknowledgments}

This paper is partially supported by CERNET Innovation Project (No. NGII20160505), National Natural Science Foundation of China (No. 61762074, No.91847302, No. 61563044 and No. 61862053) and National Natural Science Foundation of Qinghai Province (No. 2019-ZJ-7034, No. 2015-ZJ-725, No.2017-ZJ-902).

\section{References}

[1] Cai Lu.The Enlightenment of German solar power industry development[D].JiLin university,2010.(In Chinese)

[2] Prediction and analysis of solar-powered water-heater monitoring system data based on time series algorithm[J].ACTA ENERGIEA SOLARIS SINICA,2010,31(11):1413-1418.

[3] PARK, GI JU, KWEON, YOUNG BOK, CHOI, JOUNG NAE. APPARATUS AND METHOD FOR POWER GENERATION DIAGNOSIS OF SOLAR PHOTOVOLTAIC GENERATION MONITORING SYSTEM[P]. :WO2014119822,2014-08-07.

[4] S. A. Bogachev,V. V. Grechnev,S. V. Kuzin,V. A. Slemzin,O. I. Bugaenko,I. M. Chertok. On the processing and analysis of the data of the CORONAS-F/SPIRIT and other solar experiments[J]. Solar System Research,2009,43(2).

[5] PARK, GI JU, KWEON, YOUNG BOK, CHOI, JOUNG NAE. APPARATUS AND METHOD FOR POWER GENERATION DIAGNOSIS OF SOLAR PHOTOVOLTAIC GENERATION MONITORING SYSTEM[P].:WO2014119822,2014-08-07.

[6] Kalogirou,Soteris.Building integration of solar renewable energy systems towards zero or nearly zero buildings[J].International Journal of Low-Carbon Technologies.2015,10(4):379-385.

[7] Trieb, Franz.Concentrating on solar power in a trans-mediterranean renewable energy co-operation[J].European Space Agency.2004,99-108.

[8] Benson, ChristopherL.On improvement rates for renewable energy technologies: Solar PV, wind turbines, capacitors, and batteries[J].Renewable Energy.2014,p745-751.

[9] Zhang Guojing,Wang Xiaoying.Design and Implementation of the Data Sharing Platform of a Photovoltaic Power Station over IPv6.ICECS 2018.2018. 